\title{
Le plateau à thé à l'épreuve du creuset marocain : histoire,
}

\section{fabrication et usages}

\section{Baptiste Buob}

\section{Résumé}

Cet article entend contribuer à une meilleure compréhension de la manière dont le plateau, la théière et, plus généralement, les divers éléments du service à thé en métal sont devenus des objets-symboles de l'identité marocaine bien qu'empruntés récemment à l'Angleterre. L'appropriation culturelle d'objets allogènes est le résultat d'un processus complexe au sein duquel l'évolution des techniques joue un rôle essentiel. L'histoire multiséculaire des artisans dinandiers de la médina de Fès dévoile, notamment, certains aspects de la façon dont l'identité d'un peuple se construit par la faculté d'un environnement technique ancien à se transformer et à intégrer de nouveaux objets.

\section{Citer ce document / Cite this document :}

Buob Baptiste. Le plateau à thé à l'épreuve du creuset marocain : histoire, fabrication et usages. In: Horizons Maghrébins - Le droit à la mémoire, $N^{\circ} 55,2006$. Manger au Maghreb. pp. 103-113;

doi : https://doi.org/10.3406/horma.2006.2381

https://www.persee.fr/doc/horma_0984-2616_2006_num_55_1_2381

Fichier pdf généré le 05/02/2019 
Au Maroc, le the est la boisson de l'ensemble des classes sociales. Dans les cafés, les demeures ou les lieux de travail, la consommation de cette plante infusée scande, par une ritualisation plus ou moins affirmée, la temporalité marocaine. La préparation et la présentation de cette infusion obéissent à des pratiques très variées qui mériteraient à elles seules de faire l'objet de nombreuses comparaisons. On constate cependant une certaine unité: lorsqu'il accompagne la visite d'amis ou de parents, le service du thé est encore souvent effectué par le chef de famille, seule personne habilitée à conduire la cérémonie ou à désigner la personne qui aura l'honneur de saisir la poignée de la théière.

Le plateau, sur lequel reposent verres et théière, est l'ustensile qui donne la stabilité au cérémoniel. Il est aussi, comme par conduction, un symbole de pouvoir. Et sa place dans le foyer n'est pas réductible à celle de simple ustensile de service. Emblème de luxe et de réussite, le plateau en cuivre, en maillechort ou en argent a longtemps été un bien indispensable pour qui voulait faire montre d'une bonne image de soi; il est d'ailleurs devenu un élément de décoration intérieure pour toutes les classes sociales, comme peuvent l'être des pièces d'orfèvrerie.

Ayant acquis une place centrale dans l'espace domestique marocain, le plateau s'en est détaché pour occuper d'autres espaces de socialité: la siniyya ${ }^{1}$ a largement débordé l'univers gastronomique qui lui était assigné à l'origine. Le plateau compte sans aucun doute parmi les objets associés initialement à l'alimentation qui s'en est le plus affranchi, acquérant un statut unique dans la société marocaine.

Lors de cérémonies de mariage, il était d'usage, il y a encore quelques décennies, d'utiliser les grands plateaux circulaires en cuivre pour porter la mariée jusqu'à l'assemblée des convives. Les adeptes des confréries religieuses utilisent souvent un plateau pour supporter la théière, les verres, les aspersoirs d'eau parfumée à la fleur d'oranger et l'encensoir d'où s'échappe la fumée qui embaume l'atmosphère des cérémonies. Par sa seule présence, la siniyya participe à l'éloignement des "gens du monde invisible" repoussés, diton, par les propriétés magiques du cuivre ${ }^{2}$.

Le plateau est aussi musical et poétique. Les verres ou les couverts peuvent également abandonner, un temps, l'univers culinaire pour former, par un contact répété avec le plateau, un rythme régulier accompagnant chants et instruments de musique. Élevée au rang d'une métaphore désignant le peuple marocain dans son ensemble par le célèbre parolier du groupe de musique Nass El

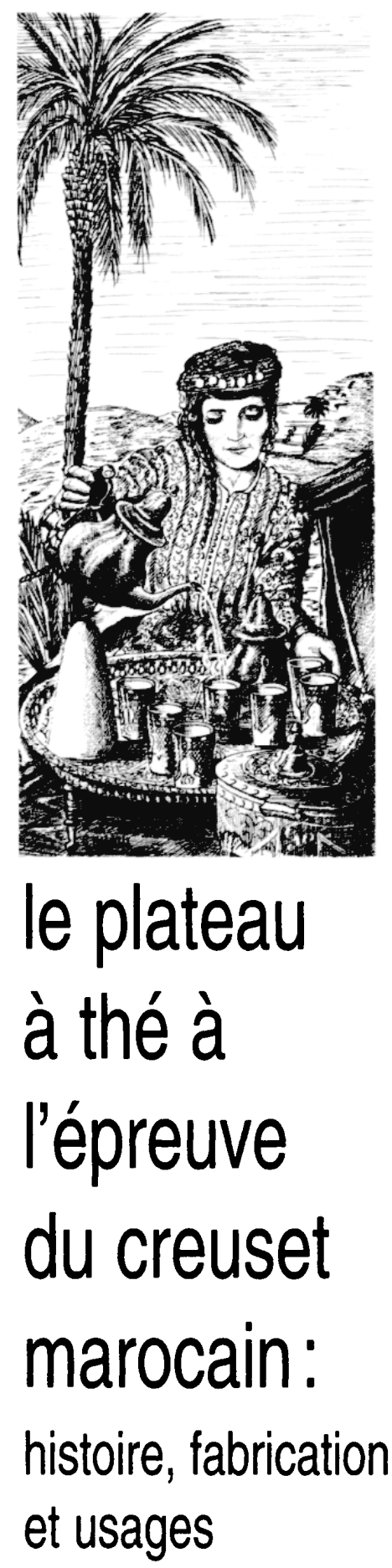

baptiste buob 
Ghiwane, Boujma', la siniyya est depuis longtemps l'objet de calligraphies musicales et poétiques, confirmant la charge émotionnelle qui la lie à nombre de Marocains.

\section{SENS DES OBJETS ET PROCESSUS D'APPROPRIATION}

Mais le terme siniyya associe différentes histoires dans un même objet. Sont mêlés, au niveau de la sémantique et des représentations, le cabaret, large plateau faisant office de table, qui se fabrique au Maroc depuis près d'un millénaire, et le plateau, spécifiquement destiné au service du thé, dont l'introduction est bien plus récente. Ce rapprochement s'est opéré, il y a près de deux siècles, en concomitance avec la diffusion du thé dans la société marocaine: la généralisation de la consommation du thé infusé et de l'utilisation de la vaisselle qui l'accompagne (plateaux, théières, verres, boîtes à sucre et à thé) y est le résultat de l'expansion du capitalisme marchand européen. L'histoire économique du thé au Maroc est connue, sa consommation ne s'y est généralisée voici guère plus d'un siècle ${ }^{3}$. Mais les ressorts culturels ayant présidé à son appropriation par la population marocaine, la manière dont une boisson à l'origine étrangère est devenue celle de tout un peuple ${ }^{4}$, sont en revanche inexplorés.

Jean-Louis Miège met en avant la "force de l'imitation" qui a poussé la masse des Marocains à reproduire des pratiques aristocratiques à l'origine $^{5}$. Dans le cadre de l'altération des modes de consommation marocains par les produits étrangers, Abdelhak El Khyari note que l'usage du thé a été accepté d'autant plus facilement que "les produits alimentaires [...] ne remettaient pas en cause, fondamentalement, le mode de vie marocain ${ }^{6} »$. Quant à Abdellah Hammoudi, il associe le processus $\mathrm{d}^{\prime}$ appropriation du thé à une tendance marocaine plus générale, selon laquelle l'innovation est sou- vent introduite de manière masquée: "Chaque innovation qui finit par s'imposer à tous devient ellemême une tradition dont la nouveauté doit nécessairement s'effacer ${ }^{7}$." Au détour de son analyse des structures sociales d'un canton du Haut-Atlas, Jacques Berque suggère que l'univers «de la rêverie, du jeu et de l'érrasion", auquel est associé le thé, a pu contribuer à sa diffusion dans le monde rural $^{8}$. Mais ces explications ne permettent pas de comprendre dans le détail le processus par lequel la population marocaine a adopté cette boisson.

Une meilleure connaissance de ce phénomène passe notamment par l'étude de la manière dont les ustensiles utilisés pour servir et préparer le thé ont acquis une place essentielle au Maroc. Pour bien saisir ce mouvement, il faudrait questionner le processus de transfert culturel dans toute sa dimension historique: comment se sont opérés les passages d'une culture à une autre, comment s'articulent les rapports entre les créateurs des objets, ceux qui se les approprient et le contexte socio-économique dans lequel s'inscrivent ces différents acteurs?

Je propose d'observer le parcours des plateaux marocains, à travers le temps, en décrivant la manière dont des artisans se sont appropriés leur fabrication: la majorité des ustensiles utilisés pour préparer le thé destinés au marché marocain sont fabriqués par les artisans swâiniya de la Médina de Fès, appellation qui désigne les «fabricants de plateaux ${ }^{9} »$. Au début du siècle dernier, la dinanderie ${ }^{10}$ ne représentait qu'une soixantaine de personnes. Aujourd'hui, au plus fort de la production, durant les mois estivaux, les dinandiers sont environ dix mille, dans près de six cents lieux de production de la vieille ville de Fès, à participer à la fabrication de multiples objets parmi lesquels des théières et des plateaux à thé.

À travers la présentation de l'histoire des swâiniya, cet article pose la question de savoir comment et pourquoi les objets du thé sont devenus des articles si fortement liés à l'identité marocaine 
tout en masquant leur origine européenne. Autrement dit, en reprenant Roland Barthes ${ }^{11}$ : comment la population marocaine s'est-elle appropriée les objets du thé au point qu'ils fonctionnent désormais comme le signe d'une certaine marocanité?

\section{LE CREUSET ORIGINEL}

En Afrique du Nord, la fabrication de plateaux en cuivre s'appuie sur un passé métallurgique qui commence dès la protohistoire. Deux millénaires avant l'ère chrétienne, dans la zone géographique correspondant au Maroc actuel occupée par les Berbères, prend forme un âge du bronze ${ }^{12}$. Premières populations à côtoyer les Berbères, les Juifs participent largement à la diffusion et à l'élaboration de savoir-faire métallurgiques - entre leur arrivée, il y a plus de deux mille ans, et leur départ, qui s'amorce au milieu du $\mathrm{Xx}^{\mathrm{e}}$ siècle, les juifs jouent un rôle déterminant dans la métallurgie marocaine, le travail des métaux étant en bonne partie dénigré par les musulmans ${ }^{13}$. Certainement attirés par les mines marocaines, les Phéniciens puis les Romains y pratiquent le moulage, comme en atteste l'abondance d'objets de cuivre et de bronze découverts sur les sites antiques ${ }^{14}$. Des géographes arabes $d u X^{\mathrm{e}}$ siècle témoignent d'une exploitation des mines - notamment de celles de cuivre de l'actuel Haut-Atlas et du Sous - et d'un commerce bien établi de métaux transformés avec l'Afrique subsaharienne ${ }^{15}$.

En Orient, avant le départ des premiers Arabes vers l'actuel Maroc, l'industrie du cuivre se développe à partir des legs persans et mésopotamiens ${ }^{16}$. Les affinités formelles et décoratives des objets métalliques du Maghreb et d'Orient suggèrent qu'à partir de cette région se diffusent les plateaux qui inspireront les artisans maghrébins. En Afrique du Nord, les Idrissides (808-930) introduisent l'usage des cuivres dans le domaine des arts mobiliers en transportant l'apparat au cœur de la vie quotidienne ${ }^{17}$. Mais $c^{\prime}$ est surtout sous les Almoravides (1055-1147) et les Almohades (1130-1269) que l'art métallurgique s'y épanouit. Durant leurs règnes, puis au début de celui des Mérinides (1269-1417), l'influence artistique orientale se diffuse au Maroc via l'Andalousie où abondent les objets métalliques d'usage courant et de luxe ${ }^{18}$. Les influences andalouses dans l'art marocain sont considérables, non seulement grâce aux réfugiés qui charrient avec eux capitaux, culture et connaissances, mais aussi par le biais des artisans qui traversent la Méditerranée pour participer à des chantiers maghrébins ${ }^{19}$. Les apports venus d'Ifriqiya, moins connus que ceux venus $d^{\prime}$ Andalousie, furent également importants. La ville de Fès est d'ailleurs composée, dès son origine, de deux quartiers, celui des Andalous et celui des Kairouanais, témoignant de la présence de populations issues du pourtour méditerranéen.

Fondée entre la fin du viII siècle et le début du IX $X^{e}$ siècle, Fès occupe une place essentielle dans le cycle métallurgique marocain: sous la dynastie almohade, le cuivre travaillé à Fès est expédié vers l'Afrique subsaharienne et, dans une moindre mesure, vers l'Europe. Cependant, en regard d'autres métiers traditionnels, le travail des métaux cuivreux n'est guère implanté à Fès - au temps des Almohades, les douze établissements fassis où l'on travaille le cuivre représentent peu à côté des 372 meules à grain, des 3064 métiers à tisser, des 47 fabriques de savon, des 86 tanneries ou encore des 116 teintureries ${ }^{20}$. Il faut dire, si l'on en croit Ibn Khaldoun, qu'une partie du travail de dinanderie était un "art inutile $[. .$.$] destiné à produire le superflu$ [...] une sorte d'agrément dû au luxe et à la richesse ${ }^{21}$ ".

Les historiens connaissent bien les objets qui attestent l'ancienneté de la dinanderie et la dextérité de ses artisans: portes de bronze, aiguières, lustres, plateaux, vases pour mesurer l'aumône légale, etc. ${ }^{22}$ Ces pièces cependant sont rares, car, 
dans la métallurgie, les artisans fondent régulièrement $d^{\prime}$ anciens objets pour en confectionner de nouveaux. La plus ancienne trace $d^{\prime}$ un plateau connu au Maroc (datant probablement du XII ou du XIII siècle) est d'ailleurs un fragment qui a été retiré d'un creuset avant sa fonte totale ${ }^{23}$.

Au Maroc se développe la fabrication d'objets en métal qui, par leurs formes et leurs usages, s'apparentent aux ustensiles du thé. La bouilloire et l'aiguière sont proches de la théière. Le cabaret oriental est très comparable au plateau à thé. Les ustensiles du thé, lorsqu'ils arriveront au Maroc, profiteront de cet usage déjà ancien de la vaisselle métallique.

Progressivement, le Maroc perd son rôle de charnière commerciale. Les contacts avec l'Orient deviennent plus rares, tandis que les relations $d u$ Maroc avec l'Afrique subsaharienne s'étiolent en raison des circuits commerciaux maritimes qui accompagnent la traite négrière européenne; jusqu'au $X v^{e}$ siècle, le cuivre représente néanmoins une part importante des exportations marocaines vers l'Afrique subsaharienne. Le divorce entre l'Europe et le Maroc s'amorce avec la fin de la Reconquista et le début du règne mérinide; en conséquence, la réciprocité de leurs relations commerciales s'atténue. L'exportation du cuivre marocain vers les pays européens subsiste, mais, de plus en plus fréquemment, les commerçants du nord de la Méditerranée se déplacent pour venir chercher le métal à sa source. Ils délaissent l'achat du métal déjà transformé au profit de minerais et ne se contentent plus d'acquérir des objets, mais en exportent au Maroc ${ }^{24}$. Les cuivres marocains transformés se vendent encore hors du Maroc à la fin du XIXe siècle ${ }^{25}$, même si, de plus en plus, les objets manufacturés en Europe concurrencent les productions locales.

Avant que les produits européens ne mettent définitivement à mal l'industrie marocaine au début du $x x^{c}$ siècle, les objets issus du nord de la Méditerranée sont parfaitement assimilés par les artisans maghrébins. Le Dictionnaire des bijoux de l'Afrique du Nord de $19066^{26}$, mentionne, à l'article "Seni", la confection au Maroc de plateaux destinés au marché algérien et l'importation de plateaux en cuivre allemands. Les Européens écoulent au Maghreb des objets qui s'inspirent des produits locaux. Tels des brûle-parfums qui proviennent d'Italie - le travail italien étant même, selon l'auteur de ce dictionnaire, plus soigné que celui des artisans algérois - ou des aiguières à long col exportées d'Allemagne. Et les fabricants maghrébins transfèrent des éléments des objets européens dans leur propre production: la décoration maghrébine de certains aspersoirs s'inspire de l'école italienne du XVII siècle, tandis que d'autres suggèrent, par leur forme, l'influence du style Louis XVI. De même, les garnitures de quelques miroirs sont des copies sur bois du travail d'ornemanistes du Midi de la France.

La métallurgie marocaine est donc un espace de rencontre entre le fond originel berbère, les influences du sud en provenance d'Afrique noire et celles de l'Orient avec les Arabes - lesquelles, déjà nourries de savoir-faire antérieurs, se sont enrichies d'emprunts européens dans le creuset andalou. Puis, progressivement, les influences viennent surtout d'Europe. À la manière d'un alliage qui mêle harmonieusement divers métaux pour obtenir une matière homogène, la dinanderie marocaine est le produit de la fusion en une même entité de multiples influences exogènes.

\section{LA VAISSELLE ANGLAISE S'INVITE À LA TABLE MAROCAINE}

Les nouveaux traités définissant les règles du commerce international - l'acte d'Algésiras (1906) en particulier - favorisent l'invasion définitive $d u$ marché marocain par des objets manufacturés étrangers: " [...] cotonnades anglaises, soieries de 
Lyon, draps de France et d'Allemagne, argenterie de Manchester, verrerie de Bohême, vaisselles et ustensiles de fer battu ou de tôle émaillée ${ }^{27}$ ». Tandis que les autorités marocaines sont devenues incapables de contrôler les dynamiques économiques ${ }^{28}$, les producteurs européens cherchent à concurrencer l'ensemble de la production locale. Au début du $x x^{e}$ siècle, il est conseillé aux Français désireux d'écouler leurs produits à Fès de copier les objets vendus sur place "sans viser à faire solide et durable, mais clinquant et d'apparence résistante ${ }^{29} \ggg$. Au nom de l'«esprit de tradition», censé caractériser les Marocains, on incite les commerçants européens à se procurer des échantillons d'articles en usage dans le pays qui serviront de modèles à imiter. Cette situation commerciale porte préjudice à la majorité des métiers marocains. Mais, grâce à la croissance du marché national des ustensiles du thé, la dinanderie y échappe en partie.

De nombreuses photographies du début du $\mathrm{xX}^{\mathrm{e}}$ siècle témoignent de la présence des ustensiles utilisés pour la préparation et le service du the dans les demeures marocaine ${ }^{30}$. On observe que les théières sont disposées de préférence sur des plateaux anglais en argent; indice de l'attachement rapide des Marocains riches pour la vaisselle métallique que l'Angleterre exporte vers le pays: "Théières en étain ou en argent; bouilloires à thé en cuivre rouge; chandeliers en cuivre jaune; cuivre rouge non travaillé en feuille; tasses à thé en terre émaillée de diverses couleurs et or; plateaux en argent pour le the et le service des repas; samovars en cuivre jaune; cuvettes en argent et leurs aiguières d'argent; plats de formes diverses en fer émaillé blanc ${ }^{31}$.»

La généralisation de cette vaisselle en métal est guidée non seulement par des agents de commerce européens mais aussi par certains Marocains $^{32}$. L'argenterie Wright (entrée dans le vocabulaire marocain sous le terme rayt) qui se diffuse dans les demeures marocaines bourgeoises en est un exemple éloquent: un commerçant marocain, installé à Manchester, entreprend, en association avec l'argentier Richard Wright, de fabriquer des articles de luxe destinés au marché marocain. Plateaux, théières, boîtes à thé, boîtes à sucre, bouilloires, brûle-parfums, plateaux garnis de lance-parfums, samovars, grands garde-pains mêlent les décorations anglaises aux répertoires des dinandiers de Fès ${ }^{33}$.

Les Européens qui diffusent la vaisselle métallique au Maroc, associés ou non à des agents de commerce marocains, cherchent à masquer l'origine des produits, tout au moins combinent-ils les goûts étrangers à la mode locale. Arrivant au Maroc, les ustensiles du thé anglais, souvent dotés d'estampilles en arabe, se délestent des attributs qui les auraient désignés trop ouvertement comme issus de l'étranger.

Le processus d'inscription du the et de ses ustensiles dans le quotidien du Maroc s'est opéré, grosso modo, en deux temps. C'est d'abord dans l'élite qu'ils se diffusent. Au cours du xvirI siècle, les missions diplomatiques étrangères venant au Maroc ont pour habitude d'apporter du thé et des ustensiles dans leurs bagages en vue de les offrir, parmi d'autres présents, aux plus hauts représentants de l'autorité marocaine ${ }^{34}$. La consommation populaire, qui constitue le second temps de la diffusion, va s'inspirer des usages aristocratiques ${ }^{35}$. Consommer le thé, c'est donc imiter les classes aisées, accéder à une pratique initialement réservée aux notables marocains et non pas copier l'étranger. Le passage préliminaire des ustensiles utilisés pour servir le thé par les classes aisées marocaines dissimule de facto son origine étrangère, d'autant plus que celle-ci est souvent masquée.

\section{L'INVENTION DU «STYLE INDIGÈNE»}

Le Maroc se trouve donc totalement cerné par l'influence du capitalisme marchand européen. L'introduction du thé et l'accaparement du mar- 
ché par des produits manufacturés ont de grandes répercussions sur les modes alimentaires des Marocains et sur la corporation des dinandiers. "Les Anglais ont réussi à communiquer à tout un peuple le goût d'une boisson étrangère, le thé, qui se confectionne avec une plante venue de Chine, du sucre venu d'Europe; on chauffe l'eau dans des bouilloires de cuivre faites à Manchester, dans des theières d'étain anglais, qu'on pose sur des plateaux dont la fabrication en arrive, elle aussi, à ne plus être marocaine. C'est un symbole ${ }^{36}$..."

Le protectorat français (1912-1956) se pose comme le garant de l'entretien d'un artisanat soustendu par des savoir-faire orientaux et andalous hérités au long des siècles. Infatigable observateur de l'artisanat marocain et acteur représentatif de la politique du protectorat dans ce domaine, Prosper Ricard déplore l'influence néfaste des produits d'importation européens et propose de "ramener les artisans dans la voie des saines traditions" en les orientant vers la production d'objets de luxe ${ }^{37}$. Un paradoxe se trouve au cœur de l'action du protectorat: la relance de savoir-faire ancestraux, considérée comme la seule manière de garantir la pérennité de l'artisanat, est pensée comme une innovation. Ce paradoxe se manifeste explicitement lorsque certains auteurs affirment la nécessité de réintroduire les techniques et les motifs d'influence andalouse ou perse pour faire évoluer l'art marocain ${ }^{38}$. La prospérité d'artisans fabriquant des objets anciens, lesquels symbolisent une authenticité fantasmée, offre le cadre exotique "indispensable» à la stratégie économique «utile» du protectorat ${ }^{39}$. Comme le dit le général Lyautey: "Où vient un peintre vient le touriste, où vient un touriste vient un homme d'affaires ${ }^{40}$."

Entravant le processus d'incorporation des influences exogènes au cœur de la dynamique artisanale (tout au moins de la dinanderie) et faute de proposer des débouchés durables pour des artisans suspendus à la demande des populations aisées, cette politique n'offre pas de pers- pectives à long terme. Dans un premier temps, malgré un contexte difficile pour l'ensemble de l'artisanat de Fès, les dinandiers travaillant pour les notables et les touristes bénéficient d'une embellie. Mais l'attrait de la clientèle pour les objets de luxe en cuivre et en laiton s'essouffle. La société marocaine s'oriente davantage vers l'acquisition d'articles produits par les industries étrangères. L'attachement à une idée exotique de ce que doit être l'artisanat contribue à figer la pratique de dinandiers qui observent, impuissants, la désagrégation des structures corporatives ayant permis, jusqu'alors, de réguler la profession.

Quand les Français arrivent au Maroc, au début du $\mathrm{Xx}^{\mathrm{e}}$ siècle, les corporations marocaines sont encore structurées par l'institution musulmane de la hisba ayant pour attribution de contrôler le respect des règles commerciales et productives ${ }^{41}$. Outre l'assurance de la qualité des productions, l'organisation corporative garantit le regroupement spatial des artisans et le contrôle de leur nombre. Elle impose le respect du travail probe et entretient la concurrence loyale. Toutefois, en contrepartie, cet esprit corporatiste, associé au conservatisme social, freine les dynamiques d'innovation. Le protectorat ne détruit pas cette institution, mais contribue à restreindre son champ d'action ${ }^{42}$.

À côté des dinandiers qui persistent dans la fabrication d'objets de luxe, d'autres se détournent de la voie tracée par le protectorat, diversifient la gamme de leurs produits et mettent en place les bases d'une industrie de substitution aux importations européennes. Pour eux, la désagrégation de la corporation est un bienfait. Avec elle s'évanouissent les rigidités de localisation, de régulation de l'innovation et de contrôle de l'effectif qui interdisaient toute refonte des manières de produire. Ces dinandiers, n'en déplaise aux tenants de l'artisanat d'art du protectorat, n'hésitent pas à charger leurs creusets d'anciennes pièces de qualité pour fondre des objets mieux 
adaptés à la demande locale. Ils renouent ainsi avec la tendance à l'alliage, au cœur du travail des métaux depuis des millénaires.

Tandis qu'une partie de la dinanderie s'accroche à une conception de la profession vacillante, l'autre s'engage donc dans une tentative de réforme du métier. Les dinandiers empruntant la voie de la rénovation entreprennent de fabriquer des articles pour la consommation de thé copiés des modèles fabriqués en Europe. Spécialisés dans la fabrication d'objets en laiton pour la consommation du thé, ils sont à l'origine de la corporation des swâiniya qui apparaît à Fès vers 1910. Avant elle, la dinanderie regroupait deux spécialités: à côté des dinandiers dits seffâra (de sffâr, le laiton ou cuivre jaune), orientés vers la confection d'éléments mobiliers (portes, lustres, plateaux, aiguières, etc.), se trouvaient les nhayisya (de $n \underline{h} \hat{a} s$, le cuivre rouge), spécialisés dans la fabrication des ustensiles de ménage en cuivre rouge (chaudrons, seaux, marmites, etc.). Ces deux corporations de la ville cohabitaient sur la place Seffarine et ses alentours. Aujourd'hui, les chaudronniers que l'on appelle encore nhayisya y officient toujours. En revanche, le terme seffâra a totalement disparu au profit de celui de swâiniya, témoignage de l'importance croissante prise par ces derniers au long $d u x{ }^{e}$ siècle - les rares artisans qui ont continué à fabriquer de produits luxueux composent désormais la maigre catégorie des dinandiers de renom fabriquant des pièces de grande valeur pour de riches commanditaires. Les swâiniya acquièrent rapidement leur autonomie et prennent une place croissante dans la Médina.

\section{LA VAISSELLE ANGLAISE, UNE PRODUCTION MAROCAINE}

Durant la dernière décennie du protectorat français apparaissent les premiers indices d'une division du travail et d'une mécanisation. Après avoir observé, lors d'un voyage en Tunisie, un Italien utiliser un tour mécanique, un dinandier introduit cette machine à Fès en $1946^{43}$. Le tour apporte de grands changements techniques et fournit les conditions favorables à la production en série d'objets autrefois importés. Grâce à lui, l'entreprise Et Taj copie très fidèlement les théières Wright fabriquées à Manchester: la théière marocaine reprend alors la forme, les décorations et les détails de la théière anglaise ${ }^{44}$. On note cependant une différence majeure: les motifs de la théière anglaise sont effectués grâce à des moules, tandis que ceux de la théière marocaine sont ciselés et poinçonnés à la main - la dinanderie perpétue la ciselure à la main des décorations (technique moins onéreuse et plus souple que celle de l'emboutissage à la presse), une pratique qui donne le cachet "traditionnel" aux objets. Certaines phases du processus de fabrication se mécanisent: le polissage (meuleuse), le planage de finition (laminoir), l'ajourage (emporte-pièce) et le plaquage (galvanoplastie).

À l'aube de la Seconde Guerre mondiale, la concurrence des produits industriels européens empêche les swâiniya de prendre la place de la grande industrie. Les dinandiers luttent pourtant contre l'emprise anglaise : conjointement à la mécanisation, ils travaillent de nouvelles matières (le maillechort, l'étain), utilisent de nouvelles techniques (la fonte par empilement, la soudure) et s'adaptent à la demande populaire (ajout de pieds aux anciens plateaux ${ }^{45}$ ).

Profitant de l'affaiblissement - voire de la destruction - des sociétés européennes exportatrices de produits de dinanderie pendant la guerre, les swâiniya prennent le contrôle d'une production d'origine exogène. Pendant que la guerre sévit en Europe, la conjoncture économique s'améliore à Fès. La désagrégation des corporations met un terme au conservatisme de l'artisanat, et les premiers éléments d'une mécanisation permettent 
aux dinandiers d'occuper le marché laissé vacant par les industries européennes en crise. Mais une des conséquences directes de la désagrégation des structures corporatives est un renforcement du rôle joué par les entrepreneurs et les intermédiaires dans le circuit de production: les artisans effectuant de leurs mains la transformation des objets sont progressivement privés des capacités d'accès à la matière première et deviennent de simples façonniers. Une minorité de dinandiers, dotés d'importantes capacités monétaires grâce auxquelles ils acquièrent matière première et outils de production, exercent progressivement une mainmise sur l'ensemble de la profession.

Avec la mécanisation et la spécialisation du travail, l'organisation de la production change. Dès le milieu des années 1960, les multiples opérations nécessaires à la confection des objets s'effectuent dans des ateliers différents: "Ainsi, un artisan dinandier découpe les éléments d'un sucrier qui seront gravés par un autre, soudés par un troisième, polis par un quatrième $e^{46}$." Alors que les ateliers produisaient autrefois toute une gamme d'articles, chaque atelier se spécialise dans la confection d'un type d'objet particulier: fabrication de plateaux pour l'un, services à thé pour un autre; d'autres encore se spécialisent dans la fabrication de lance-parfums, de lave-mains, de théières, etc.

Dans la Médina apparaissent des «unités intégrées", comparables aux fabriques de la première industrialisation en Europe, où des dinandiers devenus salariés s'affairent sur des machines. Ainsi se développent deux types d'espaces de production: d'un côté, les unités intégrées plus ou moins mécanisées où s'effectuent différentes phases du processus de transformation et, d'un autre, des ateliers dominés par l'activité manuelle où les dinandiers travaillent "à façon" en vue de l'accomplissement $d$ 'une phase particulière $d u$ processus.

$\mathrm{Du}$ fait de cette "industrialisation non orthodoxe ${ }^{47}$ ", à cheval sur l'artisanat et l'industrie, la dinanderie en est venue à mêler différentes organisations du travail dans un mode de production hybride, lequel laisse peu de place aux anciens savoir-faire et au travail manuel. Cependant, grâce à ses décorations ciselées, restées fidèles aux arabesques et aux entrelacs, la dinanderie continue à faire montre d'une inscription dans le passé «ancestral» du Maroc.

\section{LA DINANDERIE ET SES OBJETS, TRADITIONNELS MALGRÉ EUX}

Confrontés à l'arrivée massive de jeunes dinandiers issus de la campagne, nombre d'artisans héritiers d'une tradition familiale (les seffâra du début du siècle) abandonnent leurs ateliers: soit ils font faillite, le marché étriqué du luxe ne leur permettant plus de dégager des bénéfices suffisants; soit ils refusent de suivre les swâiniya, dont le travail, perdant sa propension artistique, s'apparente de plus en plus à celui de plombiers ou de zingueurs. S'ajoute à ces départs de la profession celui des juifs qui avaient toujours été très actifs dans la dinanderie. Vidée en bonne partie de ses anciens artisans, la dinanderie, qui, je le rappelle, est passée de quelques dizaines de membres au début du $x x^{e}$ siècle à plusieurs milliers aujourd'hui, perd progressivement les détenteurs de la mémoire de la profession. Les nouveaux venus dans le métier, embauchés comme ouvriers parcellisés, n'acquièrent plus aucun référent historique. S'efface alors la connaissance de l'origine anglaise de certains objets.

Au sortir du protectorat, le Maroc réaffirme ses racines orientales, forme de "retour aux sources" en réaction aux décennies de domination française. Cet état d'esprit, renforcé par la volonté de contribuer au développement économique du pays nouvellement indépendant, permet de comprendre l'attitude de la population 
marocaine qui revend sa vaisselle anglaise pour acquérir des objets produits localement. Se glisse alors le dernier voile du masquage de l'origine étrangère du thé et de ses ustensiles.

Aujourd'hui, dans le prolongement de la conception muséographique de l'artisanat instaurée sous le protectorat, la ville de Fès est encore présentée comme une ville refuge, un espace où certaines traditions ancestrales ont été mises «en conserve ". Par leur seule présence, qui suffit à les inscrire dans l'ancestralité, les swâiniya participent de l'âme de Fès; d'ailleurs, par son organisation spatiale et le discours de ses artisans, la dinanderie valorise la référence à la tradition et masque les mutations socio-économiques qui se sont fixées dans la profession depuis des décennies ${ }^{48}$. En conséquence, les objets confectionnés par les artisans sont imprégnés de la même tonalité ancestrale. Comme si la dinanderie fassie, perçue comme ancestrale, ne pouvait qu'engendrer des objets eux-mêmes ancestraux.

\section{Notes}

1. Aujourd'hui, le terme siniyya désigne un plateau en métal. Ce mot a pu qualifier de la vaisselle de service (soucoupe, table, gamelle, cabaret) - voire de cuisson (tourtière) - qui était généralement en porcelaine ou en cuivre (le terme şiny, signifie littéralement "chinois", dont dérive le terme siniyya, a pu désigner la porcelaine et des métaux cuivreux), même si ces plats pouvaient être en bois ou en cuir; Dozy Reinhart, Supplément aux dictionnaires arabes [1881], (3' éd.), t. I, Maisonneuve et Larose, Paris, 1967, p. 857-858.

2. CHEBel Malek, Dictionnaire des symboles musulmans, Albin Michel, Paris, 1995, p. 128-129. Sur les propriétés magiques des métaux en Afrique du Nord, voir également: Doutre Edmond, Magie et Religion dans l'Afrique du Nord [1908], J. Maisonneuve - P. Geuthner, Paris, 1994.

3. "À la fin du XVIII" siècle et au début du XIX siècle [la conquête du Maroc par le thél avait gagné la classe makhzen et les plus riches, de 1830 à 1860 , l'usage s'était répandu dans les milieux citadins cependant que de 1860 à 1878 , il touchait la campagne proche; de 1880 à 1892, enfin, il se répandait largement dans tout le monde rural, cernant les tribus berbères et une partie des tribus de la montagne. Dès les années 18931896 , le thé, à peu près partout apprécié, sera de plus en plus consommé; la montagne en faisant un emploi quasi général dans les premières années du siècle." MiEGE Jean-Louis, "Origine et développement de la consommation du thé au Maroc", Bulletin économique et social du Maroc, XX, 71, 1956, p. 390.

4. Les cas d'emblèmes d'une identité culturelle empruntés récemment à d'autres cultures sont courants. Sur ce type d'emprunt, avec l'exemple du thé à la menthe au Maroc, voir: BAYART Jean-François, L'Illusion identitaire, Fayard, Paris, 1996.

5. MIEGE, op. cit., p. 397.

6. EL KHYARI Abdelhak, Capitalisme et Artisanat au Maroc, 19501980, thèse de doctorat d'État en sciences économiques, faculté de droit, Rabat, 1983, p. 290.

7. Hammoudi Abdellah, Maitres et Disciples. Genèse et fondements des pouvoirs autoritaires dans les sociétés arabes [1977], Maisonneuve et Larose - Éditions Toubkal, Casablanca, 2001, p. 60.

8. Berque Jacques, Les Structures sociales du Haut-Atlas, PUF, Paris, 1955, p. 37.

9. Le terme swâiniya (sing. swâini) est issu du pluriel du mot plateau: swâni (sing. siniyya). Les deux termes swâiniya et siniyya ont bien la même racine SNY (sad - nun - ya) - la transcription du terme swâiniya se réfère à la prononciation fassie

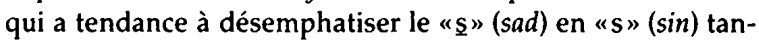
dis que celle du terme siniyya conserve l'emphatisation du "s": "s" (sad).

10. Le terme "dinanderie", dont la première occurrence remonte, en Europe, au XIII siècle, est formé à partir du nom d'une ville de Belgique, où, dès le Haut Moyen Âge, fleurit l'art du laiton: Dinant; Toussaint Jacques (dir.), Art du laiton. Dinanderie, Société archéologique de Namur, Namur, 2005. La première attestation de l'emploi de ce terme pour qualifier les artisans de Fès qui façonnent le cuivre et ses dérivés date de 1924; RiCARD Prosper, "Les métiers manuels à Fès", Hespéris, IV, 1924, p. 205-224. Dès lors, "dinandier" est couramment employé dans les travaux en français sur Fès pour désigner la catégorie marocaine de travailleurs appelés localement swâiniya. La traduction la plus fidèle de ce dernier terme est l'expression "fabricants de plateaux». Pour autant, le travail du swâini ne se limite pas à la confection de plateaux, mais consiste en la pratique d'un ensemble de techniques de mise en forme (coulage, martelage, pliage, découpe, torsion, etc.), d'assemblage (soudure) et de décoration (gravure, ciselage, estampage, etc.) de métaux et d'alliages non précieux aboutissant à la fabrication d'ustensiles ménagers et de mobilier accessoirement décorés: vaisselles, luminaires, portes, etc.

11. Barthes Roland, "Sémantique de l'objet" [1966], in Roland Barthes, L'Aventure sémiologique, Le Seuil, Paris, 1985, p. 249-260.

12. Pour un travail récent sur l'Âge du bronze au Maroc, voir: RODRIGUE Alain, L'Art rupestre du Haut-Atlas marocain, L'Harmattan, Paris, 1999.

13. Sur l'histoire des Juifs au Maroc, voir notamment: ZaFRANI Haïm, Deux mille ans de vie juive au Maroc. Histoire et culture, religion et magie, Maisonneuve et Larose, Paris, 1983. Sur l'apport spécifique des Juifs au travail des métaux marocains, voir: EL KOUTBIA Souaad, La Contribution des bijoutiers juifs à la bijouterie artisanale du Maroc du X siècle à la première moitié $d u X X^{r}$ siècle, thèse de doctorat, université Paris-VIII, 1992.

14. Pour une présentation des artefacts métalliques de la période anté-romaine découverts sur le littoral marocain, voir: ARHARBI Rachid, "L'occupation du littoral du Maroc à l'époque pré-romaine", in Caroline Gaultier-Kurhan (dir.), Patrimoine culturel marocain, Maisonneuve et Larose, Paris, 2003, p. 57-101. Pour la période romaine, voir: BOUBE-PICCOT Christiane, Les Bronzes antiques du Maroc, 4 vol., Etudes et travaux d'archéologie marocaine, IV-1961, V-1975, VIII-1980, Rabat 
et Édition recherche sur les civilisations, Paris, 1994. Pour la période entre le départ des Romains et l'arrivée des Arabes, voir notamment: RulHMANN Armand, "Moules à bijoux d'origine musulmane", Hespéris, XXI, 1935, p. 141-148.

15. Sur l'histoire de la métallurgie au Maroc, voir notamment: Colin Georges S., "Les mines marocaines et les Marocains ", Bulletin économique et social du Maroc, III, 13, 1936, p. 194-200; JodIN André, "Les gisements de cuivre du Maroc et l'archéologie des métaux", Bulletin d'archéologie marocaine, VI, Rabat, 1966; RosenberGer Bernard, "Les anciennes exploitations minières et les centres métallurgiques au Maroc. Essai de carte historique", Rerue de géographie du Maroc, 17, 1970a, p. 71-108 et «La vie des anciennes exploitations et la place des mines et des métaux dans l'histoire du Maroc", Revue de géographie du Maroc, 18, 1970b, p. 59-102; Golvin Lucien, "Note sur l'industrie du cuivre en Occident musulman au Moyen Âge", Cahiers de linguistique, d'orientalisme et de slavistique, 1-2, 1973, p. 117-126; LOMBARD Maurice, Études d'économie médiévale II. Les Métaux dans l'ancien monde du v' au Xr siècle, Mouton, Paris, 1974.

16. Pour des travaux sur l'art métallurgique en Orient, voir notamment: MIGEON Gaston, Manuel d'art musulman. Arts plastiques et industriels ( $2^{2}$ éd.), 2 t., A. Picard, Paris, 1927; BAER Eva, Metalworks in Medieval Islamic Art, State University of New York Press, Albany, 1983; MAYER Leo Ary, Islamic Metalworkers and Their Works, Genève, 1985; WARD Rachel, Islamic Metalwork, British Museum Press, London, 1992.

17. MIQcel André, L'Islam et sa Civilisation, Armand Colin, Paris, 1977, p. 308.

18. Dufource Charles-Emmanuel, La Vie quotidienne dans l'Europe médiévale sous domination arabe, Hachette, Paris, 1978; Levi-Provençal. Évariste, L'Espagne musulmane au X' siècle. Institutions et vie sociale [1932], Maisonneuve et Larose, Paris, 1996.

19. Sur les relations artisanales entre l'Andalousie et le Maroc, voir notamment: LE TOURNEAU Roger, Fez avant le protectorat. Étude économique et sociale d'une ville de l'occident musulman, Institut des hautes études marocaines, XLV, Casablanca, 1949; DeVERDUN Gaston, Marrakech, des origines à 1912, Éditions techniques nord-africaines, Rabat, 1959; BOUKOBZA André, La Poterie marocaine, Alpha - GEAM, Casablanca, 1974. 20. El IDRISSI, in Le TOLRNEAU, op. cit., p. 57.

21. IBN KHALDUN, Discours sur l'histoire universelle. Al Muqaddima [1377], traduction Vincent Monteil, Sindbab, Paris, 1978, p. 776 .

22. Sur les anciens objets cuivreux du Maroc, outre les travaux déjà cités, voir notamment: BEL Alfred, Notes sur trois anciens vases de cuivire gravé trouvés à Fès et servant à mesurer l'aumône légale du fitr, Imprimerie nationale, Paris, 1918; RICARD Prosper, Pour comprendre l'art musulman en Afrique du Nord et en Espagne, Hachette, Paris, 1924; CAMBaZARD-AmaHAN Catherine, La Décoration sur bois dans l'architecture de Fès. Époques almoravide, almohade et début mérinide, Éditions du CNRS, Aix-en-Provence, 1989.

23. DelPy Alexandre, "Note sur un coffret, une lampe et un fragment de plateau du musée régional des Arts et du Folklore de Rabat", Hespéris, LXV, 1958, p. 167.

24. Pour de précieux indices sur le commerce du cuivre entre l'Europe et le Maroc au XVII' siècle, voir: CASTRIES (de) Henry, Les sources inédites de l'histoire du Maroc, dynastie filalienne, $4 \mathrm{t}$., Ernest Leroux, 1922, 1924, 1927 et 1931.

25. ReNE-LECLERC Charles, "Le commerce et l'industrie à
Fès", Bulletin du comité de l'Afrique française, 7-8-9, 1905, p. 245. 26. Eudel Paul, Dictionnaire des bijoux de l'Afrique du Nord, Ernest Leroux, Paris, 1906.

27. MATHIAS Jean, "L'artisanat marocain", Bulletin économique et social du Maroc, XXVII, 96-97, 1963.

28. EnNaJI Mohammed, Expansion européenne et Changement social au Maroc (XVI-XIX' siècles), EDDIF, Casablanca, 1996.

29. ReNE-LECLERC, op. cit., p. 230.

30. Jacqlier Philippe, Pranal Marion et Abdelouahab Farid, Le Maroc de Gabriel Veyre (1901-1936), Kubik Éditions, Paris, 2005; KeSSAR RajI Noufissa, L'Art du thé au Maroc, ACR Édition, Paris, 2003.

31. RENE-LECLERC, op. cit., p. 239.

32. La participation des commerçants marocains, notamment fassis, au processus d'emprunt et de diffusion d'objets étrangers a sans aucun doute été essentielle tout au long des siècles. Au début du $x x^{*}$ siècle, ils entretiennent un commerce actif avec l'Algérie, l'Allemagne (Hambourg), l'Angleterre (Manchester et Londres), l'Égypte (Le Caire), la France (Lyon), l'Italie (Gènes), le Moyen-Orient, le Sénégal ou encore le Soudan; BEN ALI Driss, "Un exemple de transition: Fès au XIX" siècle", colloque "Dépendance et problématiques de la transition", du 10 au 12 avril 1980, université Mohammed V, Rabat, 1980.

33. SEBTI Abdelahad, "Itinéraires du thé à la menthe", in Collectif, Tea for Two. Les rituels du thé dans le monde, Crédit communal, Bruxelles, 1999, p. 141-153.

34. MiEGE, op. cit. et SEBTI, op. cit.

35. La population marocaine a-t-elle simplement cherché à s'approprier des objets qu'elle avait l'occasion d'observer lors de festivités aristocratiques? Des personnes au service des classes aisées ont-elles transporté dans leur demeure de tels objets? Les mécanismes précis de cet emprunt sont encore à explorer.

36. ROSENBERGER (1970b), op. cit., p. 95.

37. RICARD Prosper, "La région de Fès à l'Exposition francomarocaine de Casablanca ", in Bureau des renseignements de Fez-Ville, Fès, 1915, p. 23.

38. DeLPEY Geneviève, "Orientation de l'artisanat au Maroc", La Géographie, LXV, 1936, p. 277.

39. Sur l'instauration d'une séparation dualiste entre le "Maroc indispensable" et le "Maroc utile" sous le protectorat français, voir notamment: RIVET Daniel, Le Maghreb à l'épreune de la colonisation, Hachette, Paris, 2002; JOHENNSEN, "Tradition et modernité, catégories idéologiques des sciences sociales", Lamalif, 71,1975 , p. 38-45. Sur la politique de fossilisation exotique du protectorat, voir notamment: CHIKHAOUI Saïd, Politiques publiques et Société. Essai d'analyse de l'impact des politiques publiques sur l'artisanat au Maroc, Publications de la faculté des lettres et des sciences humaines, 54, Rabat, 2002. 40. MATHIAS, op. cit., p. 70.

41. Pour un travail de synthèse récent sur l'ancienne organisation des corporations marocaines, voir: ZIRARI-DEVIF Michèle, "La hisba au Maroc: hier et aujourd'hui", in Hervé Bleuchot (dir.), Les Institutions traditionnelles dans le monde arabe, Karthala - IRFMAM, Paris, 1996, p. 71-85.

42. Sur ce point, voir: MINER Horace, «Le déclin des corporations de Fès vu sous l'angle de la théorie des conflits", Bulletin économique et social du Maroc, XXX, 109, 1968, p. 81-94.

43. HolsSEl Jean-Pierre, "L'évolution récente de l'activité industrielle de Fès", Rev'ue de géographie du Maroc, 9, 1966, p.59-84. 
44. Sur l'histoire des formes et des motifs des théières anglaises, voir notamment: GARIBALDI Christopher, «Histoire de la théière et du thé en Grande-Bretagne ", in Collectif, Tea for Two. Les rituels du the dans le monde, Crédit communal, Bruxelles, 1999, p. 227-233.

45. Berque Jacques, "Pour le paysan et l'artisan indigène" [1939], Le Maghreb entre deux guerres, Le Seuil, Paris, 1962.

46. HOUSSEL, op. cit., p. 61.

47. Fellal Ali et Guerraouı Driss, «Fès: une industrialisation non orthodoxe ", Informations et Commentaires, 77, 1991.

48. ВСов Baptiste, "Être et paraître authentique. Tradition et identité chez les dinandiers de Fès", Cahiers de recherche du centre Jacques Berque, 3, Rabat, 2005, p. 161-183.

Résumé : Cet article entend contribuer à une meilleure compréhension de la manière dont le plateau, la théière et, plus généralement, les divers éléments du service à thé en métal sont devenus des objets-symboles de l'identité marocaine bien qu'empruntés récemment à l'Angleterre. L'appropriation culturelle d'objets allogènes est le résultat d'un processus complexe au sein duquel l'évolution des techniques joue un rôle essentiel. L'histoire multiséculaire des artisans dinandiers de la médina de Fès dévoile, notamment, certains aspects de la façon dont l'identité d'un

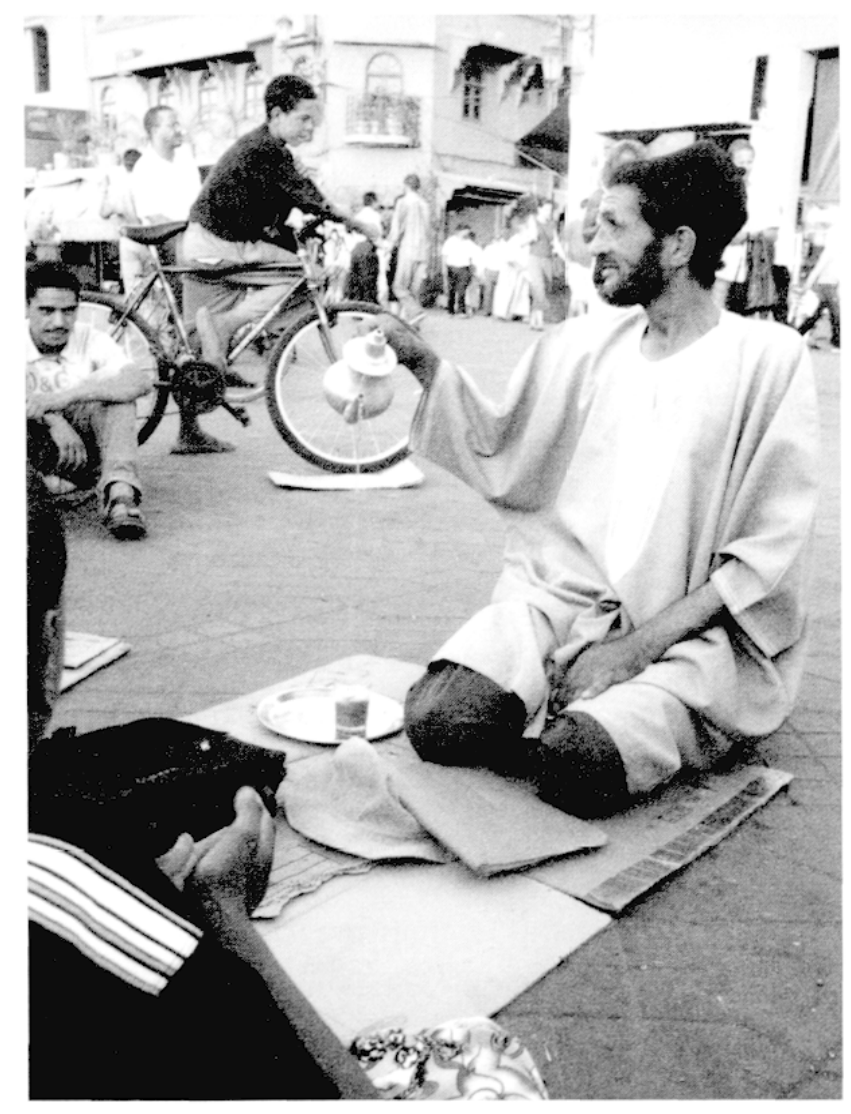

peuple se construit par la faculté d'un environnement technique ancien à se transformer et à intégrer de nouveaux objets.

Auteur: Baptiste Buob est post-doctorant de la Formation de Recherches Cinématographiques de l'université Paris 10 - Nanterre. Depuis 2000, il mène une enquête ethnofilmique sur les techniques employées par les artisans dinandiers de la médina de Fès (Maroc). Mêlant la description vidéographique de processus de fabrication d'objets en laiton à l'analyse ethnologique d'une profession, son travail interroge la notion d'«artisanat traditionnel» en la confrontant aux données historiques, économiques, techniques et sociales.

Mots clés: Maroc, Métallurgie du cuivre, Dinanderie, Plateau, Service à thé, Anthropologie des techniques, Industrialisation de l'artisanat, Construction de l'identité, Emprunts et appropriation d'objets.

Conteur de la Place Jemaa el Fna, août, 2006 photo: Mohammed Habib Samrakandi "Le thé au lieu de descendre dans mon ventre, va droit dans ma tête».

"Installé dans sa parole face au barrad (la théière), celui qui parle est comme un artisan: il cisèle les mots et les colore. Il s'oublie dans les détails, s'égare dans les couleurs écarlates, dans les choses inessentielles. Car toute chose devant lui est décorée à l'extrême: la ssinia (plateau), le barrad, les verres." Tiré du texte du poète marocain Abdallah Zrika: Le Maroc dans une théière. In Petites proses, éd. L'Escampette, 1998, Bordeaux, p. 31. 